\title{
Clinical Study of Quyuhuazhuo on Reducing Blood Hypercoagulability of Malignant Tumors
}

\author{
Aixia Sui ${ }^{1, ~ *, ~ W e n h u a ~} \mathrm{Xu}^{2}$, Huayue Cong ${ }^{1,4}$, Huiling Song ${ }^{1,4}$, Yong Liu ${ }^{5}$, Haibo Jia ${ }^{3}$, \\ Yongqing Shen ${ }^{2, *}$ \\ ${ }^{1}$ Department of Oncology, Hebei General Hospital, Shijiazhuang, China \\ ${ }^{2}$ College of Nursing, Hebei University of Chinese Medicine, Shijiazhuang, China \\ ${ }^{3}$ Department of Traditional Chinese Medicine, Hebei General Hospital, Shijiazhuang, China \\ ${ }^{4}$ College of Postgraduate, North China University of Technology, Tangshan, China \\ ${ }^{5}$ Department of Oncology, Baoding First Central Hospital, Baoding, China \\ Email address: \\ allen_sui77@sina.com (Aixia Sui),shyq2005@sina.com (Yongqing Shen) \\ ${ }^{*}$ Corresponding author
}

\section{To cite this article:}

Aixia Sui, Wenhua Xu, Huayue Cong, Huiling Song, Yong Liu, Haibo Jia, Yongqing Shen. Clinical Study of Quyuhuazhuo on Reducing Blood Hypercoagulability of Malignant Tumors. Cancer Research Journal. Vol. 7, No. 4, 2019, pp. 125-128. doi: 10.11648/j.crj.20190704.11

Received: August 28, 2019; Accepted: September 16, 2019; Published: September 27, 2019

\begin{abstract}
Objective: To investigate the clinical significance of Quyuhuazhuo on reducing blood stasis syndrome of malignant tumors (hypercoagulable state of blood). Methods: 60 malignant tumor patients with hypercoagulable state were randomly divided into treatment group and control group. In treatment group, 30 patients were treated with Quyuhuazhuo combined with chemotherapy. In control group, the other 30 patients received chemotherapy only. The clinical symptoms were observed. The changes of PLT (platelet), FIB (fibrinogen), PT (prothrombin time), APTT (activated partial thromboplastin time) and D-dimer were monitored dynamically before and after treatment. Result: The time of PT and APTT was prolonged significantly in treatment group compared with control group $(P<0.05)$, and the value of PLT, FIB and D-dimer decreased significantly $(P<0.05)$. Conclusion: The combined treatment of chemotherapy with Quyuhuazhuo can reduce the hypercoagulable state in malignant tumor patients.
\end{abstract}

Keywords: Quyuhuazhuo, Malignant TUmor, Blood Hypercoagulability, Blood Stasis

\section{Introduction}

Malignant tumors are a serious threat to human health [1]. According to the latest data, mortality of malignant tumor accounts for $23.91 \%$ of all deaths, and has worsened in the past decade. So, how to prolong the survival time of patients with malignant tumor and improve the quality of life has become an urgent problem to be solved [2]. At present, many studies indicated patients with advanced tumor have a general hypercoagulable state. The thromboembolism occurs in $5 \%-10 \%$ of tumor patients, and hypercoagulable state is an important factor in the death of cancer patients [3]. Therefore, anticoagulant therapy for patients with malignant tumor has received more and more attention. Traditional Chinese Medicine (TCM) has been widely used for disease prevention and management, and is gaining popularity worldwide for promoting health care [4]. Medical research showed that TCM can improve the body microcirculation and reduce the body's hypercoagulable state [5]. The purpose of this study is to investigate the effect of TCM Quyuhuazhuo in improving blood stasis syndrome (high coagulation state) of malignant tumor.

\section{General Information and Methods}

\subsection{General Information}

A total of 60 patients with malignant tumor were hospitalized from April 2013 to January 2014. They were randomly divided into treatment group and control group. In treatment group, 30 patients were treated with Quyuhuazhuo combined with conventional chemotherapy. In control group, 
30 patients received only chemotherapy.

\subsection{Diagnostic Criteria}

\subsubsection{Diagnostic Criteria for Blood Stasis Syndrome}

The blood stasis syndrome is determined according to clinical diagnostic criteria formulated by Chinese Society of Integrated Traditional Chinese and Western Medicine.

\subsubsection{Diagnosis of Hypercoagulable State of Blood}

According to the National Clinical Laboratory Procedures (Fourth Edition) published in 2015, One or more abnormalities such as the function of blood vessel wall and endothelial cells, the number and function of platelets, platelet metabolites, platelet antibodies, and blood coagulation factors fibrinogen, coagulation factor activation markers, anticoagulants, fibrinolysis, and fibrin degradation products can be diagnosed hypercoagulable state.

\subsubsection{Diagnostic Criteria for Malignant Tumors}

Patients were diagnosed as malignant tumor according to their clinical types, imaging and pathological cytological characteristics.

\subsection{Methods and Treatments}

Patients with malignant tumor are not allowed to use drug affected blood coagulation within 10 days after hospitalization, such as heparin, dicoumarin, aspirin, prostaglandin preparations, contraceptives, etc.

Control group: conventional chemotherapy.

Treatment group: conventional chemotherapy combined with Quyuhuazhuo. one dose a day, and taken in the morning and evening respectively. There are 5 courses of treatment (one course 10 days treatment and 5 days interval).

\subsection{Laboratory Tests}

After the enrollment, fasting venous blood was taken in the morning for laboratory tests such as platelet (PLT), fibrinogen (FIB), prothrombin time (PT), activated partial thromboplastin time (APTT), and D-dimer. After the end of treatment, the above tests were further tested.

\subsection{Statistical Methods}

The data were is analyzed using Student's t-test. The differences between two groups were considered to be statistically significant as $P$ value $<0.05$. Statistics were performed using SPSS11.0 software.

\section{Result}

The results showed that there was no significant difference in the control group before and after chemotherapy (Table 1, $P>0.05$ ), suggesting that chemotherapy had no significant effect on the hypercoagulable state of these patients. The parameters were significantly improved after chemotherapy in the treatment group and the difference was statistically significant (Table 2, $P<0.05$ ), indicating that assisted therapy of Quyuhuazhuo can reduce the hypercoagulability of patients with malignant tumor.

Table 1. Comparison of various indicators before and after treatment in the control group.

\begin{tabular}{lllll}
\hline indicators & Before treatment & After treatment & $\boldsymbol{t}$ & \multicolumn{1}{c}{ P } \\
\hline PT $(\mathrm{S})$ & $6.23 \pm 1.45$ & $6.63 \pm 1.10$ & 1.93 & $>0.05$ \\
APTT $(\mathrm{S})$ & $16.73 \pm 2.20$ & $16.47 \pm 1.59$ & 0.94 & $>0.05$ \\
FIB $(\mathrm{g} / \mathrm{L})$ & $5.59 \pm 0.83$ & $5.70 \pm 0.63$ & 1.59 & $>0.05$ \\
PLT $\left(\times 10^{9} / \mathrm{L}\right)$ & $436.97 \pm 40.94$ & $441.07 \pm 40.09$ & 1.03 & $>0.05$ \\
D-dimer $(\mu \mathrm{g} / \mathrm{L})$ & $560.07 \pm 81.56$ & $560.80 \pm 73.56$ & 0.18 & $>0.05$ \\
\hline
\end{tabular}

Table 2. Comparison of various indicators before and after chemotherapy in the treatment group.

\begin{tabular}{lllll}
\hline indicators & Before treatment & After treatment & $\boldsymbol{t}$ & \\
\hline PT $(\mathrm{S})$ & $5.83 \pm 1.34$ & $10.43 \pm 1.36$ & 16.80 & $<0.05$ \\
APTT $(\mathrm{S})$ & $16.80 \pm 2.62$ & $30.67 \pm 2.01$ & 29.11 & $<0.05$ \\
FIB $(\mathrm{g} / \mathrm{L})$ & $5.54 \pm 0.78$ & $3.82 \pm 0.27$ & 14.15 & $<0.05$ \\
PLT $\left(\times 10^{9} / \mathrm{L}\right)$ & $455.30 \pm 36.53$ & $297.27 \pm 11.90$ & 22.31 & $<0.05$ \\
D-dimer $(\mu \mathrm{g} / \mathrm{L})$ & $597.90 \pm 45.11$ & $253.47 \pm 47.43$ & 39.90 & $<0.05$ \\
\hline
\end{tabular}

\section{Discussion}

Hypercoagulability is common in patients with malignant tumor [6]. Tumor cells can damage the vascular endothelium, strengthen the adhesion, aggregation and activation of platelets, secrete procoagulant substances, reduce anticoagulant activity, inhibit the activation of fibrinolytic system, and cause hypercoagulable state [7]. It believed that balancing gas, blood, yin, yang, phlegm, and dampness has a beneficial effect on hypercoagulability of patients with malignant tumor in Chinese medicine [8]. Anticoagulant or antithrombotic therapy may improve the prognosis of tumor [9]. For hypercoagulable states, drugs block the coagulation state, such as heparin and low molecular weight heparin (LMWH). Oral direct anticoagulants can reduce hypercoagulability and reduce the number of D-dimers. These drugs may help prevent thromboembolic events in patients with cancer hypercoagulable states. Vitamin $\mathrm{K}$ antagonist and warfarin can reduce the production of coagulation factors, but those does not interrupt the coagulation cascade may not help reduce hypercoagulable state, and it will increase the risk of bleeding [10], especially cerebral hemorrhage greatly increased the mortality rate and disability rate. So, it is 
especially important for the treatment of malignant tumors with thrombosis. In addition, the hypercoagulable state of the blood is more common in the elderly. While the elderly have more basic diseases, they often taking multiple drugs at the same time, which is prone to occur drug interactions. Chinese medicine treatment has a comparative advantage. The Chinese herbal medicine formula has the advantages of "multi-component, multi-target, multi-channel", combined regulation mechanism and relatively low toxicity [11]. Chinese Medicines have been widely used in cancer treatment, such as hepatocellular carcinoma (HCC) and colon cancer [12, 13]. Chinese medicines can promote blood circulation and lower the blood hypercoagulability state to prevent cancer cells from being inhibited by anticancer drugs and autoimmune cells. They can reduce platelet aggregation and reduce tumor metastasis. It can also help anti-inflammatory and anti-infective. It can enhance the effect of chemotherapy and reduce the side effects of chemotherapy.

Quyuhuazhuo is composed of Sanling, Ezhu, Baizhuo, Shijianchuan, Baihuasheshecao, Yuanhu, Shemei, Baizhilian, and Tianjihuang. Among them, the Sanling has a bitter taste, bitterness and flatness. It can be used to break the blood clotting. The Ezhu is spicy, bitter and warm. It has also a strong clotting-breaking effect. They can enhance anticoagulation when both are used together. Baizhuo taste bitter, slightly cold, non-toxic, attending evil belly and abdominal pain, eliminating blood stasis, broken accumulation of cold and hot, and pain. The functional is nourish and soft liver, and relieve pain. Shijianchuan taste bitter, slightly cold. The function is to promote blood circulation. Baihuasheshecao is sweet, light, and cool. The functional is to clean away heat, detoxify, and eliminate phlegm. According modern research, it can enhance immune function and promote antibody formation. YuanHu bitter, warm, can promote blood circulation and disperse phlegm. Baizhilian, xin, ping, non-toxic, can clear away heat and detoxify. It can be used for cirrhosis ascites and various cancers through modern research. Tianjihuang sweet, slightly bitter, and cold. The function is clearing heat and detoxifying, eliminating dampness and reducing yellowness. It is used for acute and chronic hepatitis, early cirrhosis, liver pain, appendicitis, mastitis, lung abscess and so on. The combination of various drugs can break hypercoagulability, reduce swelling and disperse, and relieve pain, which is good for removing scorpion venom and eliminating toxins. Ezhu, Chinese herbal medicine for promoting blood circulation and removing blood stasis, has the function of promoting the normalization of endothelial cells of blood vessels of tumor-derived [14]. Thereby, it can reduce the hypercoagulable state of patients.

\section{Conclusion}

In summary, coagulation abnormalities are common in patients with malignant tumors, and the main manifestation is hypercoagulable state [15]. In this study, our data indicated that combined treatment of chemotherapy with Quyuhuazhuo can reduce the hypercoagulable state in malignant tumor patients. These results expand the clinical application range of some Chinese medicine and also provide new ideas for cancer treatment.

\section{Acknowledgements}

This work was supported Guiding Questions of the Chinese Medicine Bureau (2013163).

\section{Conflicts of interest}

All the authors do not have any possible conflicts of interest.

\section{References}

[1] Bray F, Ferlay J, Soerjomataram I, Siegel RL, Torre LA, Jemal A. Global cancer statistics 2018: GLOBOCAN estimates of incidence and mortality worldwide for 36 cancers in 185 countries. CA Cancer J Clin. 2018, 68 (6): 394-424.

[2] Blane DN, Lewandowska M. Living with cancer and multimorbidity: the role of primary care. Curr Opin Support Palliat Care. 2019, 13 (3): 213-219.

[3] Al-Samkari H, Connors JM. Dual anticoagulation with fondaparinux and dabigatran for treatment of cancer-associated hypercoagulability. Am J Hematol. 2018, 93 (6): E156-E158.

[4] Normile D. DRUG DISCOVERY. Nobel for antimalarial drug highlights East West divide. Science. 2015, 350 (6258): 265.

[5] Araújo A. [Cancer and deep venous thrombosis: the purpose of the CATCH clinical trial]. Acta Med Port. 2013, 26 (2): 83-85.

[6] Scărlătescu E, Lancé MD, White NJ, Aramă SS, Tomescu DR. Effects of malignancy on blood coagulation in septic intensive care patients. Blood Coagul Fibrinolysis. 2018, 29 (1): $92-96$.

[7] Ay C, Pabinger I, Cohen AT. Cancer-associated venous thromboembolism: burden, mechanisms, and management. Thromb Haemost. 2017, 117: 219-230.

[8] Wang S, Long S, Wu W. Application of Traditional Chinese Medicines as Personalized Therapy in Human Cancers. Am J Chin Med. 2018, 46 (5): 953-970.

[9] Al-Samkari, H; Connors, JM; Dual anticoagulation with fondaparinux and dabigatran for treatment of cancer-associated hypercoagulability. Am J Hematol. 2018, 93 (6): E156-E158.

[10] Hsu JY, Liu AB. Anticoagulants for cancer-associated ischemic stroke. Ci Ji Yi Xue Za Zhi. 2019, 31 (3): 144-148.

[11] Ling CQ, Yue XQ, Ling C. Three advantages of using traditional Chinese medicine to prevent and treat tumor. J Integr Med. 2014, 12 (4): 331-335.

[12] Xi SY, Minuk GY. Role of traditional Chinese medicine in the management of patients with hepatocellular carcinoma. World J Hepatol. 2018, 10 (11): 799-806. 
[13] Kang XJ, Wang HY, Peng HG, Chen BF, Zhang WY, Wu AH, $\mathrm{Xu} \mathrm{Q}$, Huang YZ. Codelivery of dihydroartemisinin and doxorubicin in mannosylated liposomes for drug-resistant colon cancer therapy. Acta Pharmacol Sin. 2017, 38 (6): 885-896.

[14] Zang W, Bian H, Huang X, Yin G, Zhang C, Han LI, Hao P, Ding S, Sun YU, Yang Z, Hoffman RM, Tang D. Traditional Chinese Medicine (TCM) Astragalus Membranaceus and
Curcuma Wenyujin Promote Vascular Normalization in Tumor-derived Endothelial Cells of Human Hepatocellular Carcinoma. Anticancer Res. 2019, 39 (6): 2739-2747.

[15] Campello E, Ilich A, Simioni P, Key NS. The relationship between pancreatic cancer and hypercoagulability: a comprehensive review on epidemiological and biological issues. $\mathrm{Br} \mathrm{J}$ Cancer. 2019 Jul 22. doi: 10.1038/s41416-019-0510-x. [Epub ahead of print] 